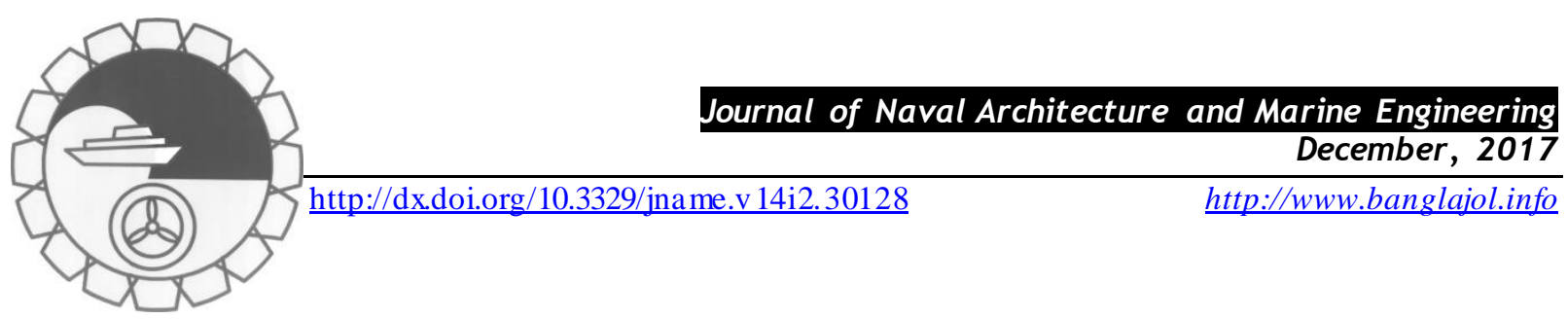

\title{
A MOLECULAR DYNAMIC STUDY OF CHANGE IN THERMODYNAMIC FUNCTIONS OF SILICON FCC CELL WITH THE CHANGE IN TEMPERATURE
}

\author{
A.B.M. Mainul Bari ${ }^{1}$, Saeed Rubaiee ${ }^{2}$, Anas Ahmed ${ }^{3}$ and A.K.M. Masud ${ }^{4 *}$ \\ ${ }^{1}$ Department of Industrial and Production Engineering, Bangladesh University of Engineering and Technology (BUET), \\ Dhaka-1000, Bangladesh, mainulbari@ipe.buet.ac.bd \\ ${ }^{2,3,4}$ Department of Industrial Engineering, University of Jeddah, KSA. \\ ² $\underline{\text { salrubaiee@uj.edu.sa, }}{ }^{3}$ aaahmed5@uj.edu.sa, ${ }^{4 *}$ MASAOD@uj.edu.sa
}

\begin{abstract}
:
In modern days silicon is being extensively used in making electronic semiconductor-based chips and $I C$ 's. Knowing silicon' thermodynamic functions are quite important, because many electronic companies are nowadays trying a lot to reduce the heat generated by their semiconductor chips. Excessive heating of the chip not only warms up the device quickly but also reduces the chip life. In this research, the change in different thermodynamic properties of silicon like lattice heat capacity, molar enthalpy and Debye temperature at constant pressure, with the change in temperature, has been investigated by using molecular dynamics (MD) simulation method. For simulation "Accelrys Materials Studio" (Version 5.0) software has been used. The simulation was run for silicon FCC diamond structured cell. The analysis tool used in the simulation is known as CASTEP (Cambridge Sequential Total Energy Package). This tool is specialized for performing molecular level thermodynamic analysis to generate data and graphs for the change in different temperature dependent properties of the molecular system. The interaction between silicon atoms was expressed by the Kohn-Sham potential and MD calculation was conducted on crystalline state of silicon at temperatures between 0 and $1000 \mathrm{~K}$. Here, density function theory (DFT) based tool has been used to derive density of state relations. Results obtained by the simulation were compared with published experimental values and it was found that the simulation results were close to the experimental values. The results obtained from this simulation will help engineers to design electronic chips more efficiently.
\end{abstract}

Keywords: molecular dynamics simulation; silicon; FCC silicon; heat capacity; enthalpy; Debye temperature, nanomaterial.

\section{NOMENCLATURE}

$\begin{array}{ll}E, S, F, C & \text { energy, entropy, free energy, and lattice heat capacity } \\ E(T) & \text { Temperature dependence of energy } \\ E_{z p} & \text { zero point vibrational energy } \\ k, h, F(\omega) & \text { Boltzmann's constant, Planck's constant, phonon density of states } \\ N, \Theta_{D} & \text { number of atoms per cell, Debye temperature } \\ v_{m} & \text { maximum frequency } \\ D_{3} & \text { third-order Debye function }\end{array}$

\section{Introduction}

Silicon is widely used in different engineering applications. It is generally found in sands and dusts in the form of silica or silicates. Silicon is widely used in different process industries such as metal process, chemical process industries etc. Highly purified silicon is often used in making electronic semiconductor to semiconductor devices, which has immense importance in current electronics industries. Pure monocrystalline silicon is also used in silicon wafers and photovoltaic application. Pure silicon also has charge conduction properties with application of heat energy. Electrical conductivity increases greatly by doping of silic on with 
other element. Thus it has application in electronic and semiconducting devices. In photonics, silicon is used to produce internal light source.

Single crystalline silicon has application in large-scale-integrated-circuit (LSI) devices, and amorphous silicon as materials for solar cells (Höhler et al 2006; Wang et al 2011). For these applications modeling for the heat and fluid flow in the crystal growth process, and modeling for thermos-physical property for crystalline and liquid silicon with high accuracy are required. Thus, this paper focuses on the molar heat capacity, enthalpy and Debye temperature at constant pressure of silicon (NPT).

Several data have been reported for molar enthalpy and heat capacity at constant pressure of silicon (Serebrennikov and Gel'd, 1952; Barin 1973; and Yamaguchi and Itagaki, 2002). According to findings of the above researchers the value of temperature coefficients are negative. However, Gerlach et al. (1972), Grove (1967) and Grazovet et al. (1969) have obtained a temperature relation is different from others. There are also considerable deviations in published data for the molar heat capacities at constant pressure of solid cryst alline silicon. It is very difficult to understand factors causing such deviations. Here, molecular dynamics (MD) simulation as a tool to measure realistic values has been considered to use. On the other hand, silicon exhibits two phases such as solid silicon in semiconductor and liquid silicon in metallic, thus application of MD in silicon becomes difficult. Furthermore, Stillinger and Weber (1985), Pavesi et al. (2010), and Ciccotti et al. (2014) have reported a potential of silicon useful for MD simulation, which enables the structure of silicon to be simulated in both the faces of crystalline and liquid states. Accordingly, it is likely that thermo-physical properties such as enthalpy, heat capacity and Debye temperature of silicon can be analyzed by using MD with no chemical contamination. Consequently, the enthalpy, molar heat capacity and Debye temperature at constant pressure of crystalline silicon has been determined using MD simulation.

\section{Related Theories}

\subsection{Force field}

In this research, Materials Studio software package has been used to perform the MD simulations and COMPASS module of the software has been selected for force-field computations. The force field can be described as average interactions among the molecules and atoms in terms of different sets of different functions and parameter in the model unit cell of the MD simulations (Tahreen and Masud, 2012). It has been seen in the earlier research works that the COMPASS force-field can be used to simulate some organic and inorganic molecules (Sun et al., 1998; Bodryakov et al., 2000). Although the used parameters for the COMPASS force field has been proprietary, it is tested and validated for some common organic and inorganic materials (Sun, 1998).

\subsection{Thermodynamic calculations}

First, thermodynamic indicators have been evaluated by the photon frequencies in Brillion zone (Kittel, 2005; Patterson et al., 2007). The results are used to find energy $(E)$, entropy $(S)$, free energy $(F)$, and lattice heat capacity $\left(C_{v}\right)$ as functions of temperature. Then, the vibrational effects in thermodynamic properties were considered to calculate $E, S, F$ and $C_{v}$ at finite temperatures.

Vibrational analysis has been done by CASTEP and during this analysis, thermodynamic calculations has been visualized using the thermodynamic analysis tools. The temperature dependence of the energy is given by (Baroni et al., 2001; Nomura et al. 2015; Liao et al. 2015):

$$
E(T)=E_{\text {tot }}+E_{z p}+\int \frac{\hbar \omega}{\exp \left(\frac{\Lambda \omega}{k T}\right)-1} F(\omega) d \omega
$$

were, $E_{z p}$ if is the zero point vibrational energy, $k$ is Boltzmann's constant, $h$ is Planck's constant and $F(\omega)$ is the phonon density of states, $E_{z p}$ can be evaluated as:

$E_{z p}=\frac{1}{2}+\int F(\omega) h \omega d \omega$

The vibrational contribution to the free energy, $F$ is given by:

$$
F(T)=E_{\text {tot }}+E_{z p}+k T \int F(\omega) \ln \left[1-\exp \left(-\frac{\hbar \omega}{k T}\right)\right] d \omega
$$

The vibrational contribution to the entropy, $S$ can be obtained using the following equation: 
$S(T)=k\left\{\int \frac{\frac{\hbar \omega}{k T}}{\exp \left(\frac{\hbar \omega}{k T}\right)-1} F(\omega) d \omega-\int F(\omega)\left[1-\exp \left(-\frac{\hbar \omega}{k T}\right)\right] d \omega\right\}$

After figuring out entropy and free energy relations, temperature dependency of enthalpy can be easily be derived.

Now, if $C_{v}$ is the lattice contribution to the heat capacity, then

$C_{v}(t)=k \int \frac{\left(\frac{\hbar \omega}{\mathrm{kT}}\right)^{2} \exp \left(\frac{\lambda \omega}{\mathrm{k} T}\right)}{\left[\exp \left(\frac{\hbar \omega}{\mathrm{k} T}\right)-1\right]^{2}} F(\omega) d \omega$

Heat capacity in Debye model is given by (Ashcroft and Mermin, 1976), which is $C_{D}^{D}(T)=9 N k\left(\frac{T}{\Theta_{D}}\right)^{a} \int_{0}^{\Theta D T} \frac{x^{4} e^{x}}{\left(e^{x}-1\right)^{2}} d x$

where, $N$ is the number of atoms per cell. Thus, the value of the Debye temperature, $\Theta_{D}$ at a given temperature, $T$, is obtained by calculating the actual heat capacity, equation (5), then inverting equation (6) to obtain $\Theta_{D}$.

\subsection{Debye temperature model}

In thermodynamics and solid state physics, the Debye model is a method developed by Peter Debye in 1912 for estimating the phonon contribution to the specific heat (heat capacity) in a solid. It treats the vibrations of the atomic lattice (heat) as phonons in a box, in contrast to the Einstein model, which treats the solid as many individual, non-interacting quantum harmonic oscillators (Debye, Peter 1912).

Debye derived an equation for calculating the number of vibrational state as shown below:

$$
n \sim \frac{1}{a} v^{\mathrm{a}} V F
$$

where $V$ is the volume and $F$ is a factor which he calculated from elasticity coefficients and density. Combining this with the expected energy of a harmonic oscillator at temperature $T$ (already used by Einstein in his model) would give an energy of

$$
U=\int_{0}^{\infty} \frac{h v^{3} V F}{e^{h v} V_{k T-1}} d v
$$

If the vibrational frequencies continued to increase to infinity, this form gives the $T^{3}$ behavior. But Debye realized that there could not be more than $3 N$ vibrational states for $N$ atoms. He made the assumption that in an atomic solid, the spectrum of frequencies of the vibrational states would continue to follow the above rule, up to a maximu $\mathrm{m}$ frequency $v_{m}$ chosen so that the total number of states is $3 \mathrm{~N}$.

$$
3 N=\frac{1}{a} v_{m}^{a} V F
$$

Debye knew that this assumption was not really correct (the higher frequencies are more closely spaced than assumed), but it guarantees the proper behavior at high temperature (the Dulong-Petit law). The energy is then given by:

$$
\begin{aligned}
& U=\int_{0}^{v m} \frac{k v^{3} V F}{d k v_{k T-1}} d v=V F k T(k T / h)^{a} \int_{0}^{T_{D} / T} \frac{x^{3}}{e^{x}-1} d x \\
& \text { where } T_{D} \text { is } h v_{\mathrm{m}} / \mathrm{k} \\
& =9 N k T\left(T / T_{D}\right)^{a} \int_{0}^{\frac{T_{D}}{T}} \frac{x^{3}}{\theta^{x}-1} d x \\
& =3 N k T D_{a}\left(T_{D}^{\infty} / T\right)
\end{aligned}
$$

where $D_{3}$ is the function later given the name of third-order Debye function.

- For Low temperature limit

The temperature of a Debye solid is said to be low if $\ll<T_{D}$, leading to

$$
\frac{C_{V}}{N k} \sim 9\left(\frac{T}{T_{D}}\right)^{a} \int_{0}^{\infty} \frac{x^{4} e^{x}}{\left(e^{x}-1\right)^{2}} d x
$$

This definite integral can be evaluated exactly: 


$$
\frac{C_{V}}{N k} \propto \frac{12 \pi^{4}}{5}\left(\frac{T}{T_{D}}\right)^{3}
$$

Note that, in the low temperature limit, the limitations of the Debye model mentioned above do not apply, and it gives a correct relationship among (phononic) heat capacity, temperature, the elastic coefficients, and the volume per atom (the latter quantities being contained in the Debye temperature).

- For high-temperature limit

The temperature of a Debye solid is said to be high if $T \gg T_{D}$ Using $e^{x}-1 \approx x$ and if $|x| \ll 1$ it leads to

$$
\begin{aligned}
& \frac{C_{V}}{N k} \sim 9\left(\frac{T}{T_{D}}\right)^{a} \int_{0}^{\frac{T_{D}}{T}} \frac{x^{4}}{x^{2}} d x \\
& \frac{C_{V}}{N k} \sim 3
\end{aligned}
$$

This is the Dulong-Petit law, and is fairly accurate although it does not take into account anharmonicity, which causes the heat capacity to rise further. The total heat capacity of the solid, if it is a conductor or semiconductor, may also contain a non-negligible contribution from the electrons.

\section{Model Construction and Simulation Condition}

The model for simulation is constructed using the graphical tools provided in the 'Materials Studio' software package, using a lattice parameter $5.43 \mathrm{~A}^{\circ}$ and a coarse Self consistent field. Standard Kohn-Sham potential (Kohn, Walter; Sham, Lu Jeu, 1965) is used to express the interaction between silicon atoms - this potential has been constructed so as to reproduce the structures of crystalline silicon just above the melting point. A unit cell is considered for MD simulation where FCC diamond structure is arranged by 19 silicon atom. Threedimensional periodic boundary condition is considered for the unit cell. MD simulation is carried out with the simulation tool provided by the software 'Materials Studio (Version 5.0)' produced by Accelrys Ltd.

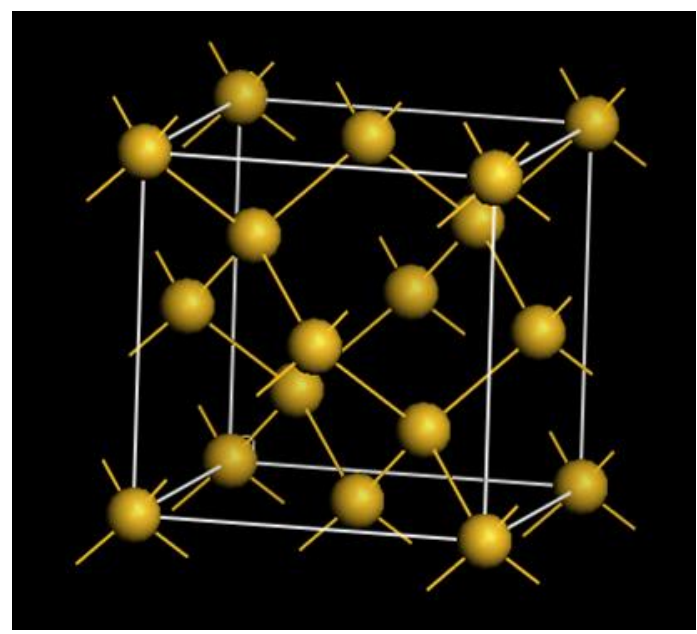

Fig. 1: Constructed unit cell for simulation

The CASTEP tool is used for analyzing thermal properties. COMPASS (Condensed-phase Optimized Molecular Potentials for Atomistic Simulation Studies) force field is used in the model for simulation, which is a specialized forcefield for analyzing organic and inorganic small molecules. The micro-canonical ensemble approach is considered for the simulation. Equations of motion are considered for the atoms in the model which are integrated with 1 fs time interval with the use of fifth-order predictor-corrector algorithm.

Is othermal-is obaric (NPT) ensemble heating is considered for the MD simulation in order to get the model of silicon structure to an equilibrium state. During calculation, heating cycle is considered between the temperature range 0 and $1100 \mathrm{~K}$ in order to stabilize the system at equilibrium. The unit cell is constructed at a temperature lower than required temperature, and constant enthalpy for more than 300ps, confirmed that the system is in 
equilibrium. The volume of the unit cell and the enthalpy and trajectories of atoms at each MD condition are determined from data obtained in the last $25 \mathrm{ps}$.

Finally, to determine the change in enthalpy and molar heat capacity with the change in temperature at constant pressure at higher accuracy, MD simulation is carried out by means of NVE ensemble using the unit cells obtained in the above. Calculation is conducted for $450 \mathrm{ps}$ at temperatures between 0 and $1100 \mathrm{~K}$ and consequently phonon density of state and phonon dispersion data for silicon is obtained, from which the data for temperature dependent change in enthalpy, Debye temperature and molar heat capacities has been derived at constant pressure.

\section{Simulation Results and Discussion}

Figure 2 shows the temperature dependencies of molar enthalpy for silicon. There are two curves in the graph. One was obtained in this simulation and other one was obtained from the research conducted by Kojima Endo et al (2003). In the curve obtained from this simulation, it is seen that, the molar enthalpy increases almost lin early, with increasing temperature up to $245 \mathrm{~K}$ and then shows another slightly nonlinear increase starting from $250 \mathrm{~K}$ up to almost $610 \mathrm{~K}$, followed by another linear increase starting from $760 \mathrm{~K}$ up to the end of the curve (1000 $\mathrm{K}$ ). The maximum enthalpy up to this point is almost $-335 \mathrm{KJ} \mathrm{mol}^{-1} \mathrm{In}$ addition, the trajectory of silicon atoms during simulation showed that the atoms moved around lattice sites at temperatures lower than 1000K. From this, we can say in this simulation silicon was in solid crystalline state the whole time, i.e., the melting point was not within, or near $1000 \mathrm{~K}$. From other experiments, melting point of silicon was found out to be at almost $1683 \mathrm{~K}$. That's why the atoms do not diffuse much from the lattice site in this simulation. Although at the simulation performed by Kojima Endo et al, for heating cycle the corresponding curve was almost linear with almost $30^{\circ}$ inclination with horizontal base, within the given temperature range. However the results from our experiment seem consistent, but slightly different from theirs with respect to the enthalpy level at the highest temperature considered in the study $(1000 \mathrm{~K})$.

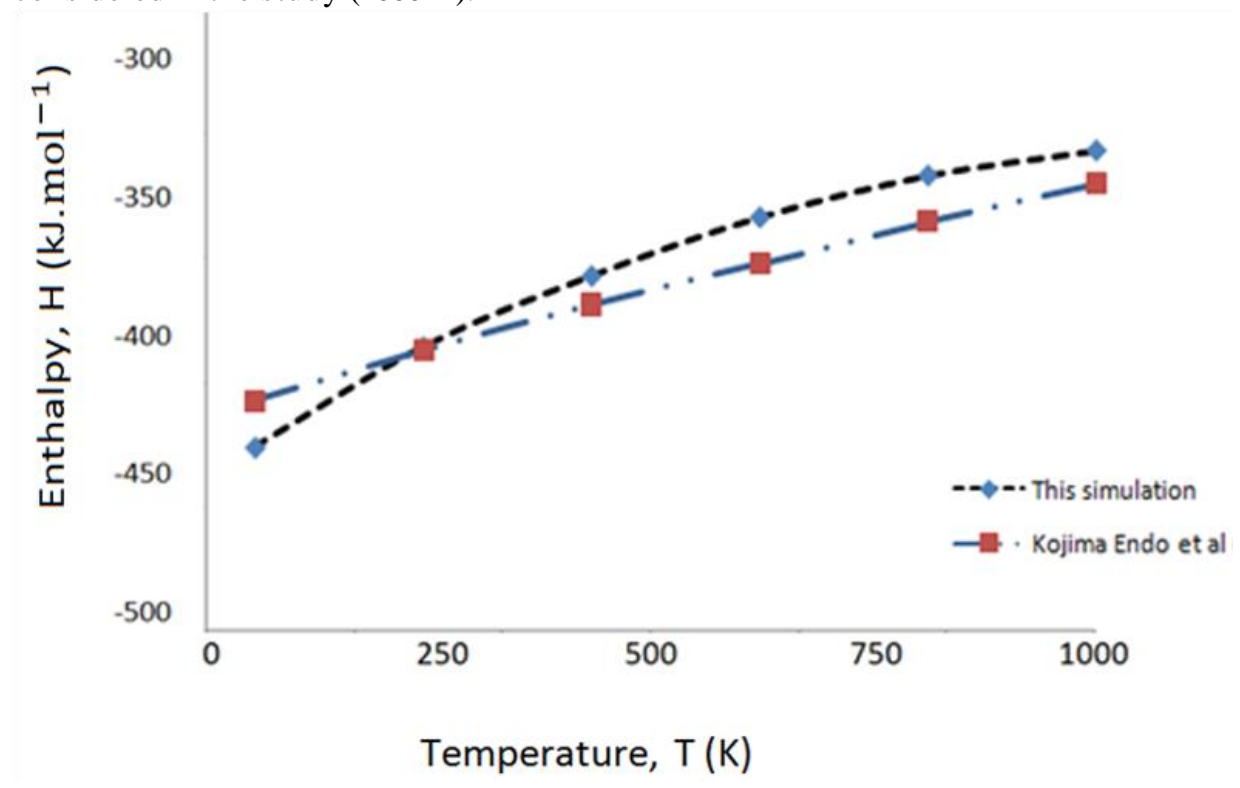

Fig. 2: Change in molar enthalpy with temperature

Heat capacity or thermal capacity is the measurable physical quantity that shows the amount of heat required to change the temperature of a substance by a given amount, where, the heat capacity per mole of a substance or the specific heat capacity, often simply called specific heat, is the heat capacity per unit mass of a material. Figure 3 shows the change in molar heat capacity at constant pressure $\left(C_{p}\right)$ for crystalline silicon as functions of temperature, in comparis on with published values (Serebrennikov and Gel'd 1952; Barin 1973; Yamaguchi and Itagaki 2002). The curve displayed by black hash-double dot indicates the curve obtained from our simulation. It shows that the molar heat capacity of silicon increases linearly from around $19.5 \mathrm{JK}^{-1} \mathrm{~mol}^{-1}$ up to $22.5 \mathrm{JK}^{-1} \mathrm{~mol}^{-1}$ when temperature of the system increases from $200 \mathrm{~K}$ to $550 \mathrm{~K}$. Later heat capacity increases in a less steep manner which also seems a little curvilinear. This trend starts around $608 \mathrm{~K}$ and continues up to the end 
temperature of the simulation $(1000 \mathrm{~K})$. During that the value of the heat capacity increases up to almost $24.2 \mathrm{~J}$ $\mathrm{K}^{-1} \mathrm{~mol}^{-1}$

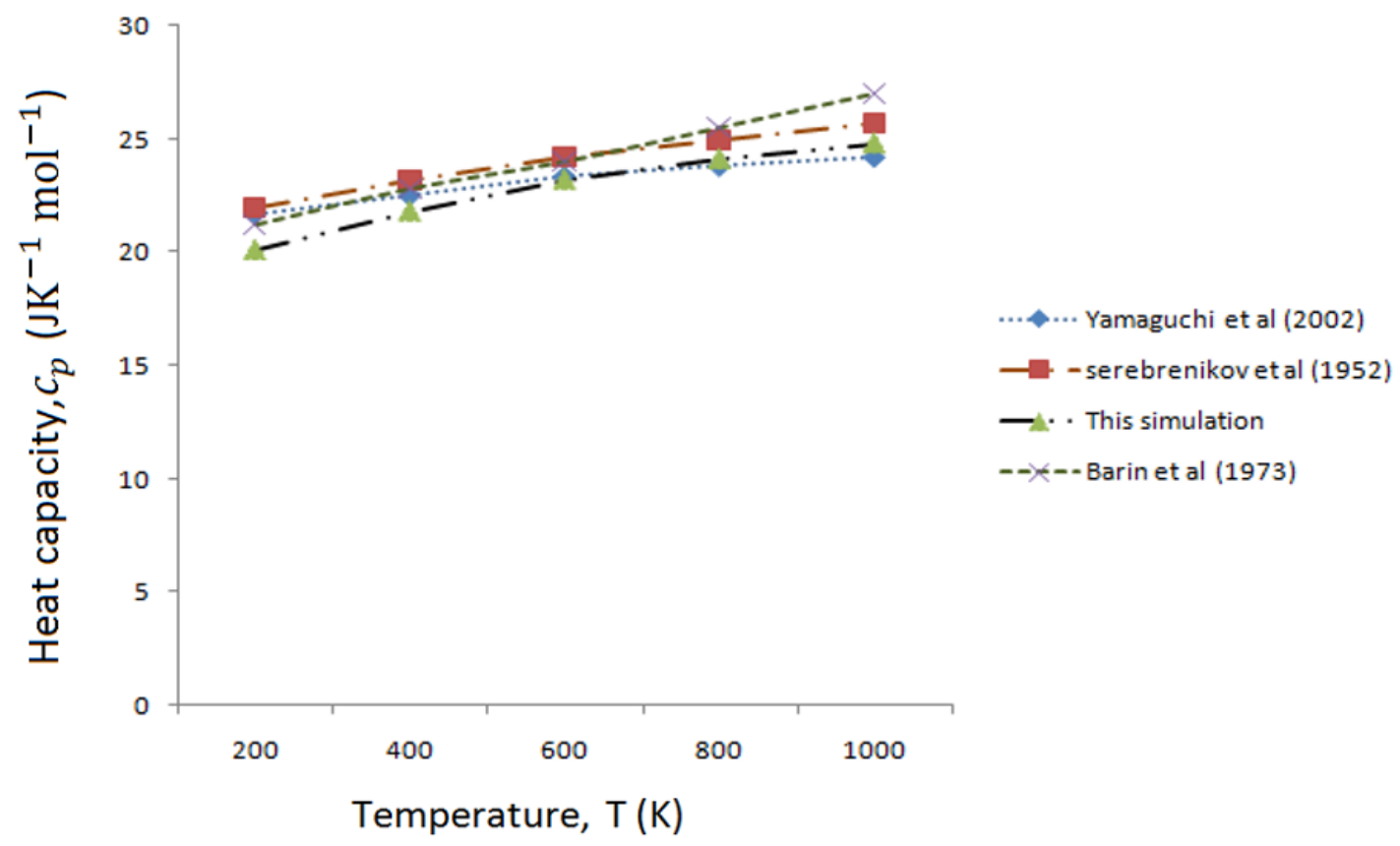

Fig. 3: Change in molar heat capacity with temperature

It appears that, the trend of change in molar heat capacity, obtained from the experiments of other scientists are a little bit different, although it's not quite unreasonable. For example, in Figure 3 the red colored hash-single dotted line indicates the curve obtained by Serebrenikovet.al. (1952). This curve is almost linear from 200 to $600 \mathrm{k}$ and again from 610 to $1000 \mathrm{k}$ with an increase in heat capacity from 22 to $25.2 \mathrm{~J} \mathrm{~K}^{-1} \mathrm{~mol}^{-1}$ at the end. Again, the gray full-hashed line (curve obtained by Yamaguchi et. al. 2002) is slightly inclined but linear up to $580 \mathrm{~K}$ and later almost horizontal from $605 \mathrm{~K}$ up to the end temperature of the simulation $(1000 \mathrm{~K})$. Therefore, we can see that their output are not really that much different from the output obtained in this research, but it does give us slightly different heat capacity with the change in temperature, thus giving a slightly different pattern of change of heat capacity.

The From the Debye model, it is found that the low temperature heat capacity is proportional to $\mathrm{T}^{3}$. Just like the Einstein model, it also recovers the Dulong-Petit law at high temperatures. But due to simplifying assumptions, its accuracy suffers at intermediate temperatures. From Figure 4, it is found, at 0 K, Debye temperature is $50 \mathrm{~K}$. Later Debye temperature increases steeply up to $410 \mathrm{~K}$, at a linear rate until the temperature is $14 \mathrm{~K}$. After that, Debye temperature varies up to $450 \mathrm{~K}$ in a relatively less steep manner and later it increases in a more steep but linear manner up to almost $600 \mathrm{~K}$. After that, Debye temperature increases up to $722 \mathrm{~K}$ as the system temperature increases to $1000 \mathrm{~K}$, although the slope during this increase was very low. Experimental results, without anharmonicity, show that the Debye temperature, at the high temperature limit is $650 \mathrm{~K}$, where our simulation obtained maximum Debye temperature is $722 \mathrm{~K}$. So we can see our obtained temperature limit is not much far from the experimental values, which justifies the correctness of our parameter selection for this experiment.

Hence, the results are quite acceptable given the level of calculation performed. The accuracy might improve further if one considers better k-points grid. It should also be noted that the quasi-harmonic approximation, on which the phonon calculation is based, becomes inefficient at temperatures higher than one-third of the Debye temperature. As a result, at temperatures higher than the Debye temperature, anharmonic effects appear that cannot be explained by a change of temperature alone. 
CASTEP Thermodynamic Properties

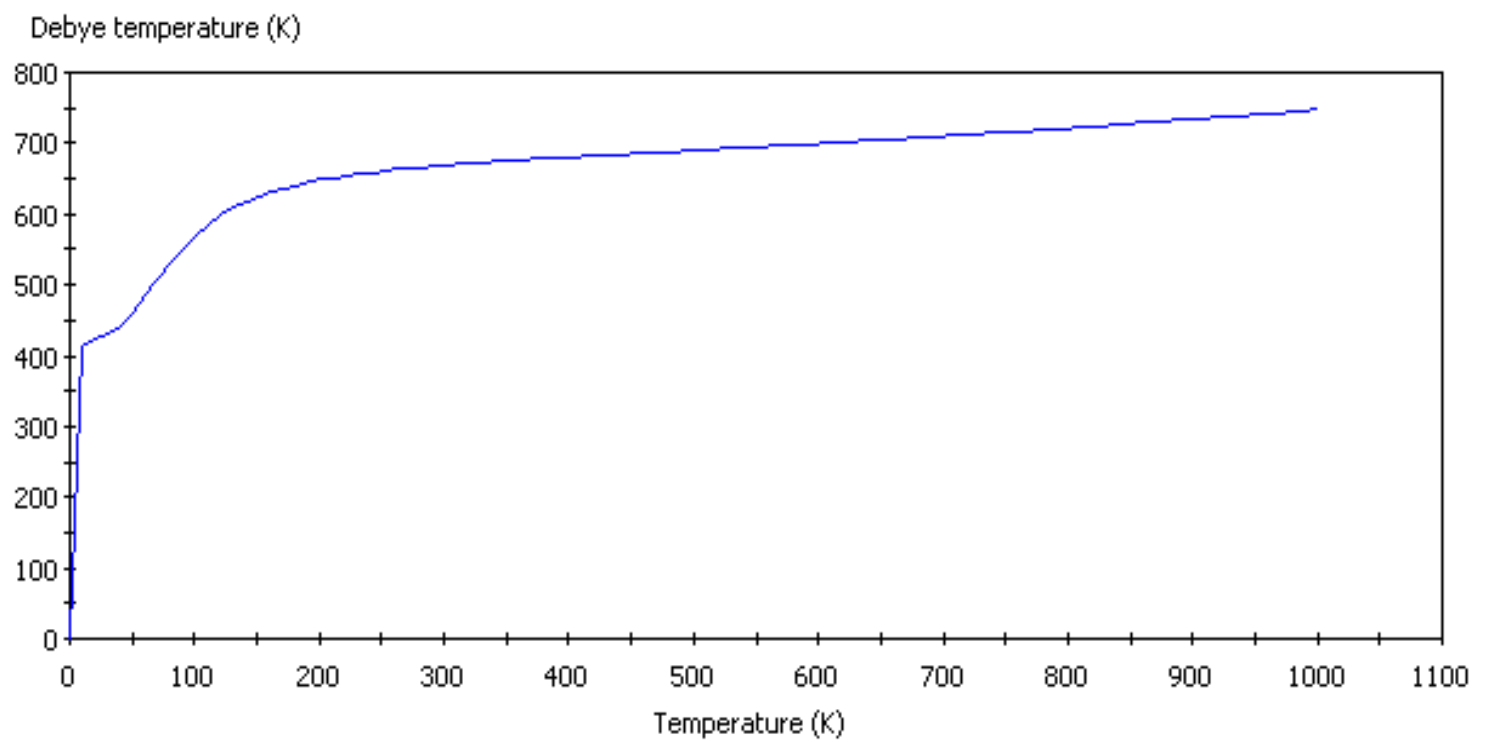

Fig. 4: Debye Temperature vs. Temperature curve for silicon

\section{Conclusions}

In our research, we studied the changes in different thermodynamic properties of silicon like lattice heat capacity, molar enthalpy and Debye temperature at constant pressure, with the change in temperature using molecular dynamics (MD) simulation method. We found out that the molar enthalpy increases almost linearly with increasing temperature up to $245 \mathrm{~K}$ and then shows another slightly nonlinear increase starting from $250 \mathrm{~K}$ up to almost $610 \mathrm{~K}$, followed by another linear increase starting from $760 \mathrm{~K}$ up to the end of the curve (1000 $\mathrm{K})$. The maximum enthalpy up to that point is almost - $335 \mathrm{KJ} \mathrm{mol}^{-1}$ Molar heat capacity of silicon increases linearly from around $19.5 \mathrm{~J} \mathrm{~K}^{-1} \mathrm{~mol}^{-1}$ up to $22.5 \mathrm{~J} \mathrm{~K}^{-1} \mathrm{~mol}^{-1}$ when temperature of the system increases from $200 \mathrm{~K}$ to $550 \mathrm{~K}$. Later heat capacity increases in a less steep manner which also seems a little curvilinear. This trend continues up to the end temperature of the simulation $(1000 \mathrm{~K})$. During that the value of the heat capacity increases up to almost $24.2 \mathrm{~J} \mathrm{~K}^{-1} \mathrm{~mol}^{-1}$. In the study it has also been found that at $0 \mathrm{~K}$, Debye temperature is $50 \mathrm{~K}$. Later Debye temperature increases steeply up to $410 \mathrm{~K}$, at a linear rate until the temperature is $14 \mathrm{~K}$. Later on Debye temperature increases up to $722 \mathrm{~K}$ as the system temperature increases to $1000 \mathrm{~K}$, although the slope during this increase was very low. The accuracy of the experiment might improve further if better k-points grid is considered.

The results and the curves obtained from this simulation have multiple applications. These can be used to predict phase stability of different molecular structural modifications like - to perform studies of phase transitions, to design new energy efficient chip from silicon that will generate less heat (thus increasing the chip's life), etc. Debye temperature results obtained from the simulation is a quite important tool as it is related to the phonon contribution to heat capacity at low temperatures. Here, it is possible to find the Debye temperature as the photon activation temperature in crystal. The Debye model gives a very good approximation for the heat capacity of insulating solid crystal. Hence, a crystal with a large Debye temperature is going to be a stiffer crystal (e.g., Diamond's Debye temperature must be larger than silicon's or copper's must be larger than lead). In this way, various important insights can be obtained from the results of this simulation.

\section{References}

Ashcroft, N. W. and Mermin, N. D. (1976): Introduction to Solid State Physics, Saunders, Philadelphia.

Barin, I., Knacke, O. and Kubaschewski, O. (1977): Thermodynamical Properties of Inorganic Substances, Supplement.

Baroni, S., De Gironcoli, S., Dal Corso, A. and Giannozzi, P. (2001): Phonons and related crystal properties from density-functional perturbation theory, Reviews of Modern Physics, 73(2), 515. 
https://doi.org/10.1103/RevModPhys.73.515

Bodryakov, V. Y. and Zamyatin, V. M. (2000): Analysis of thermodynamic functions of silicon at high temperatures, High Temperature, 38(5), 698-704. https://doi.org/10.1007/BF02755921

Ciccotti, G., Ferrario, M. and Schuette, C. (2014): Molecular Dynamics Simulation. Entropy, 16, 233.

https://doi.org/10.3390/e16010233

Debye, P. (1912): Zur theorie der spezifischen wärmen. Annalen der Physik, 344(14), 789-839.

https://doi.org/10.1002/andp.19123441404

Endo, R. K., Fujihara, Y. and Susa, M. (2003): Calculation of the density and heat capacity of silicon by molecular dynamics simulation, High Temp High Press, 35(36), 505-511. https://doi.org/10.1068/htjr135

Gerlach, W., Schlangenotto, H. and Maeder, H. (1972): On the radiative recombination rate in silicon, Physica Status Solidi (a), 13(1), 277-283. https://doi.org/10.1002/pssa.2210130129

Grove, A. S. (1967): Physics and technology of semiconductor devices, Wiley.

Höhler, G., Fujimori, A., Kühn, J., Müller, T., Steiner, F., Stwalley, W. C.... and Woggon, U. (2006): SolidState Physics, Vol. 68 Springer.

Kittel, C. (2005): Introduction to solid state physics, Wiley.

Kohn, W. and Sham, L. J. (1965): Self-consistent equations including exchange and correlation effects, Physical review, 140(4A), A1133. https://doi.org/10.1103/PhysRev.140.A1133

Liao, B., Qiu, B., Zhou, J., Huberman, S., Esfarjani, K. and Chen, G. (2015): Significant reduction of lattice thermal conductivity by the electron-phonon interaction in silicon with high carrier concentrations: A firstprinciples study, Physical Review Letters, 114(11), 115901. https://doi.org/10.1103/PhysRevLett.114.115901

Nomura, M., Nakagawa, J., Kage, Y., Maire, J., Moser, D. and Paul, O. (2015): Thermal phonon transport in silicon nanowires and two-dimensional phononic crystal nanostructures, Applied Physics Letters, 106(14), 143102. https://doi.org/10.1063/1.4917036

Patterson, J. D. and Bailey, B. C. (2007): Solid-state physics: introduction to the theory, Springer Science and Business Media.

Pavesi, L. and Turan, R. (Eds.) (2010): Silicon nanocrystals: fundamentals, synthesis and applications, John Wiley \& Sons. https://doi.org/10.1002/9783527629954

Serebrennikov, N. M. and Gel'd, P. V. (1952): Dokl. Akad. Nauk. SSSR871021.

Stillinger, F. H. and Weber, T. A. (1985): Computer simulation of local order in condensed phases of silicon, Physical Review B, 31(8), 5262. https://doi.org/10.1103/PhysRevB.31.5262

Sun, H., Ren, P. and Fried, J. R. (1998): The COMPASS force field: parameterization and validation for phosphazenes. Computational and Theoretical Polymer Science, 8(1), 229-246.

https://doi.org/10.1016/S1089-3156(98)00042-7

Tahreen, N. and Masud, A. K. M. (2012): A molecular dynamics study of the variations in the elastic properties of single-walled carbon nanotubes with tube radius, length and chirality, International Journal of Nano and Biomaterials, 4(1), 21-32. https://doi.org/10.1504/IJNBM.2012.048214

Tahreen, N. and Masud, A. K. M. (2012): Investigation of the mechanical properties of polyethylene/carbon nanotube composite by molecular dynamics simulation, International Journal of Nano and Biomaterials, 4(1), 54-68. https://doi.org/10.1504/IJNBM.2012.048217

Wang, Z., Alaniz, J. E., Jang, W., Garay, J. E. and Dames, C. (2011): Thermal conductivity of nanocrystalline silicon: importance of grain size and frequency-dependent mean free paths, Nano Letters, 11(6), 2206-2213.

https://doi.org/10.1021/nl1045395

Yamaguchi, K. and Itagaki, K. (2002): Measurement of high temperature heat content of silicon by drop calorimetry, Journal of thermal Analysis and Calorimetry, 69(3), 1059-1066.

https://doi.org/10.1023/A:1020609517891 